\title{
Functional neuroimaging: magnetoencephalography
}

\section{Erin S. Schwartz}

(C) Springer-Verlag 2011

Magnetoencephalography (MEG) is increasingly implemented as an important part of the pre-operative evaluation in the setting of intractable epilepsy. With MEG, epileptogenic zones can be non-invasively localized and the relationship to eloquent functional cortex highly accurately determined. These provide the epilepsy team with additional valuable information with which they can assess the likelihood of postoperative seizure freedom, while more precisely prognosticating the potential functional deficits that might result from resective surgery.

Paradigms for motor, vision, auditory and somatosensory testing are robust. Paradigms allowing localization of those regions necessary for competent language function, although promising, are under continuous optimization. Correlation with MR white matter trajectory data, created from diffusion tensor imaging obtained in the same setting as the localization brain MRI, provides more comprehensive, multi-modal information for the neurosurgeon. A collaborative effort among neuroradiologists, neurologists, neurosurgeons, neuropsychologists, technologists and physicists is needed for successful implementation of MEG within an epilepsy program.

MEG also provides highly accurate functional mapping that can be extremely valuable in the preoperative evaluation of intracranial neoplasms and arteriovenous malformations. Particularly given the alterations in blood flow that frequently accompany these conditions, functional mapping with MEG has been shown to be superior to functional MRI.

Disclaimer Dr. Schwartz has no financial interests, investigational or off-label uses to disclose.

E. S. Schwartz $(\bowtie)$

Children's Hospital of Philadelphia,

Philadelphia, PA 19104 USA

e-mail: SchwartzES@email.chop.edu 